\title{
Proteomic analyses on the browning of shade-dried Thompson seedless grape
}

\author{
Fengjuan Liư ${ }^{1,2}$, Wenshu Huang ${ }^{2}$, Zuoshan Feng ${ }^{2}$, Yongxia Tao ${ }^{2}$, Yingying Fan' ${ }^{1}$, Weizhong He ${ }^{1,2}$, XiaoLi Li ${ }^{1}$, \\ Xiaotong Fang ${ }^{1}$, Cheng Wang ${ }^{1,3^{*}}$ (ID and Yujia Bai ${ }^{2^{*}}$
}

\begin{abstract}
China is one of the main producers in the worldwide raisin market. Most China's raisins are produced in Xinjiang where the Thompson seedless grape (Vitis vinifera L.cv.Thompson seedless) is the main variety of green raisin. However, the browning of Thompson seedless grape during drying has been well-acknowledged as the primary factor affecting the development of the raisin industry. Data independent acquisition (DIA)-based protein profiling was performed on fresh and shade-dried Thompson seedless grapes. As a result, 5431 proteins were identified, among which the amounts of 739 proteins in fresh grape were found to be significantly different with those in dried grape. The functional annotation based on the Blast2GO showed that the 'organic substance metabolic process,', regulation of molecular function', 'enzyme regulator activity', and 'isomerase activity' related proteins became very active during browning. Further analyses revealed that the browning-related proteins, which with significant different amounts in fresh and in dried grapes, are primarily involved in the phenylpropanoid biosynthesis, tyrosine metabolism, phenylalanine metabolism, oxidative phosphorylation metabolism, plutathione metabolism, peroxisome pathway, and fatty acid degradation. And five random differential proteins were verified with parallel reaction monitoring (PRM). The PRM results were in agreement with the DIA data. The main browning-related proteins of Thompson seedless grape were identified in this study. Their properties were tested, and their roles in the browning mechanism were indicated. This will lay base to a better understanding on the enzymatic browning of Thompson seedless grape, and it will also provide guidance for controlling the quality of Thompson seedless grapes in industry.
\end{abstract}

Keywords: Thompson seedless grape, Browning, DIA, Proteomics, PRM, KEGG pathway

\section{Introduction}

Thompson seedless grape (Vitis vinifera L.cv.Thompson seedless) has been a major raisin grape variety worldwide for over a century [1]. China's raisin production accounts for about $15 \%$ of the world's total output, ranking third after the United States and Turkey. Raisins are not only delicious, but also with high nutritional values [2]. Due to

\footnotetext{
*Correspondence: wangchengxj321@sina.com; saintbyj@126.com ${ }^{1}$ Institute of Quality Standards \& Testing Technology for Agro-Products, Xinjiang Academy of Agricultural Sciences, Key Laboratory of Agro-Products Quality and Safety of Xinjiang, Laboratory of Quality and Safety Risk Assessment for Agro-Products (Urumqi), Ministry of Agriculture and Rural Affairs, Urumqi 830091, China

${ }^{2}$ College of Food Science and Pharmacy, Xinjiang Agricultural University, Urumgi 830052, China

Full list of author information is available at the end of the article
}

the low moisture content, raisins are resistant to storage and transportation.

Xinjiang, the largest raisin producing area in China, possess the output of 180,000 tons in 2018, among which $60 \%$ were green raisins. The main variety of green raisins is Thompson seedless grape [3], which is mainly produced in Turpan region of Xinjiang. Due to the superior photothermal conditions and unique climate in Turpan, shade-dry is the most common drying method used in the production of raisins. Natural dry hot air in a special four-wall ventilation is used as drying medium. The product is not directly exposed to the sunlight. The browning of the raisins prepared in this way is very common. Based on investigations, the browning rate of raisins in different regions of Xinjiang varies from 40 to $65 \%$. 
Biochemical reaction of phenolic substances caused by polyphenol oxidase (PPO) and peroxidase (POD) were considered as the primary factor for the browning of shade-dried Thompson seedless grape. In healthy fresh fruit, PPO and POD present in a soluble form in cytoplasm, while they bind to organelle membrane and cell membrane or cell wall in plastid, mitochondria, chloroplast, etc. The phenols are distributed in vacuole $[4,5]$, where enzymes cannot get access to the reaction with phenolic substances. As a result, fresh grapes remain green without browning. However, under the influence of non-biological forces such as aging and dehydration after harvest, the biofilm systems of fruits and vegetables, such as cell membrane and organelle membrane, will be damaged. Consequently, the cellular compartments could be released from their original locations, and the contact between oxidases and phenolic substances will produce enzymatic browning [6].

The shade-dry process is a type of abiotic stress for Thompson seedless grape, which can cause metabolism changes on the grape. These changes may affect the browning of grape. At present, the research on browning of Thompson seedless grape mainly focuses on the changes of polyphenol oxidase and its phenolic substances [7-10]. The proteomics changes during drying in shade of Thompson seedless grape are rarely studied. In this study, fresh Thompson seedless grape (browning index 1), browning grapes (browning index 6) were used as raw materials. The proteomics changes of Thompson seedless grape during drying in shade were studied by using data independent acquisition (DIA) proteomics technology. Systematic analysis on different proteins and metabolic pathways during browning was performed. Moreover, parallel reaction monitoring (PRM) was applied to validate the DIA results. This study provides a reference for the browning mechanism of Thompson seedless grape drying in shade.

\section{Materials and methods Chemicals and reagents}

Sucrose, Tromethane hydrochloride (Tris- $\mathrm{HCl}$ ), Ethylenediamine tetraacetic Acid (EDTA), Lauryl sodium sulfate (SDS), Phenol, Acetone, Formic acid, Potassium chloride $(\mathrm{KCl})$ were purchased from Sinopharm (Beijing, China); 1,4-Dithiothreitol (DTT), Trypsin, Bond-Breaker $^{\mathrm{TM}}$ TCEP Solution (TCEP), Iodoacetamide and Acetonitrile were purchased from Sigma-Aldrich (Johannesburg, South Africa); $1 \times$ Protease Inhibitor Cocktail was purchased from Roche Ltd. (Basel, Switzerland); iRT peptides were purchased from Biognosys (Schlieren, Switzerland).

\section{Equipments}

Refrigerated centrifuge, $-80{ }^{\circ} \mathrm{C}$ refrigerator, Orbitrap Lumos coupled to EASY-nLC 1200 system and on-line nanospray LC-MS/MS on Orbitrap Fusion ${ }^{\text {TM }}$ Lumos $^{\text {TM }}$ Tribrid $^{\mathrm{TM}}$ mass spectrometer coupled to an EASY-nanoLC 1000 system were purchased from Thermo Fisher Scientific (Massachusetts, USA).

\section{Fruit materials and treatment}

Fruit of grapes (Vitis vinifera L.cv.Thompson seedless) at commercially mature stage (Brix: $20-22 \%$ ) were harvested on 20 August 2019 from a garden located in Turpan in Xinjiang, China. They were selected for their uniformity of size (diameter: $12.51 \mathrm{~mm} \pm 0.81 \mathrm{~mm}$, length: $16.99 \mathrm{~mm} \pm 1.28 \mathrm{~mm}$ ), ground colour, and exemption from defects and mechanical damage. Then $200 \mathrm{~kg}$ of Thompson seedless grapes were kept in the shade for drying. Samples were taken every 2 days for measurements. One gram grapes for proteomic analysis were sampled from fresh grapes (FG) and browned grapes (BG). The FG samples were collected on the harvest day, and the BG samples were from the batch that had been kept at shade for drying for 30 days. All the samples were independently prepared in triplicate and stored at -80 ${ }^{\circ} \mathrm{C}$ until use.

\section{Measurement of browning index}

The browning index (BI) of Thompson seedless grape was measured according to the method described by Lin et al. [11]. Browning was assessed by measuring the extent of the browned area of 50 individual fruits, the BI was calculated as $\Sigma$ (browning scale $\times$ proportion of corresponding fruits within each class).

\section{Protein preparation}

The samples were homogenized in liquid nitrogen, and then $2 \mathrm{~mL}$ of lysis buffer ( $30 \%$ sucrose, $0.5 \mathrm{M}$ Tris- $\mathrm{HCl}$, $50 \mathrm{mM}$ EDTA, $20 \mathrm{mM}$ DTT, $0.1 \mathrm{M} \mathrm{KCl}, 2 \%$ SDS, $1 \times$ Protease Inhibitor Cocktail) was added into the samples. Cell lysis was performed by sonication on ice for 1 $h$ and followed by centrifugation at $4000 \times g$ for $30 \mathrm{~min}$ at $4{ }^{\circ} \mathrm{C} .2 \mathrm{~mL}$ of Tris-saturated phenol (pH 7.5) was added and the resulting mixture was thoroughly vortexed for $30 \mathrm{~min}$ at $4{ }^{\circ} \mathrm{C}$ and centrifuged for phase separation. The organic phase was collected for use. The extraction step was repeated once. Five volumes of acetone were added to the combined organic phase and precipitated at -20 ${ }^{\circ} \mathrm{C}$ overnight. $100 \mu \mathrm{g}$ of protein from each sample was transferred into a new Eppendorf tube and the final volume was adjusted to $100 \mu \mathrm{L} .2 \mu \mathrm{L}$ of $0.5 \mathrm{M}$ TCEP was added and the sample was incubated at $37^{\circ} \mathrm{C}$ for $1 \mathrm{~h}$, and then $4 \mu \mathrm{L}$ of $1 \mathrm{M}$ iodoacetamide was added to the sample, 
followed by an incubation in dark for $40 \mathrm{~min}$. After that, five volumes of acetone were added to precipitate the proteins overnight. Sequence grade modified trypsin was added to digest the proteins at $37^{\circ} \mathrm{C}$ overnight.

\section{Liquid chromatography-mass spectrometry analysis}

For the library generation by DDA, all 6 samples were pooled and separated into 6 fractions using high $\mathrm{pH}$ separation. The peptides were re-dissolved in solvent $\mathrm{A}$ (A: $0.1 \%$ formic acid in water) and analyzed by on-line nanospray LC-MS/MS on Q Exactive ${ }^{\text {TM }}$ HF-X coupled to EASY-n LC 1200 system. For retention time and m/z calibration, the iRT peptides were added to the peptides. Three microliter peptide sample was loaded to a trap column (ThermoFisher Scientific Acclaim PepMap C18, 100 $\mu \mathrm{m} \times 2 \mathrm{~cm}$ ), and subsequently to an analytical column (Acclaim PepMap C18, $75 \mu \mathrm{m} \times 25 \mathrm{~cm}$ ). The samples were separated by a 120 min-gradient from 5 to $60 \%$ of solvent $\mathrm{B}$ (B: $0.1 \%$ formic acid, $80 \% \mathrm{ACN}$ ). The flow rate was maintained at $400 \mathrm{~nL} / \mathrm{min}$. The electrospray voltage of $2 \mathrm{kV}$ versus the inlet of mass spectrometer was used. The mass spectrometer was run under data dependent acquisition mode. It automatically switched between MS and MS/MS mode. DIA analysis was performed by the same LC-MS/MS system as DDA.

\section{LC-MS data analyses}

Raw Data of DDA were processed and analyzed by Spectronaut 13 (Biognosys AG, Switzerland) with default settings to generate an initial target list, which contained 58,642 precursors, 41,550 peptides, 5431 proteins and 5261 protein group. Spectronaut was set up to search the Uniport database of Vitis vinifera (version 201907, 29875 entries) assuming trypsin as the digestion enzyme. Carbamidomethyl $(\mathrm{C})$ was specified as the fixed modification. Oxidation $(\mathrm{M})$ was specified as the variable modifications. The cutoff of $Q$ value (FDR) on PSM, precursor and protein level were set to $1 \%$. Raw Data of DIA were processed and analyzed by Spectronaut 13 (Biognosys AG, Switzerland) with default settings, according to the iRT peptide segment software, the retention time can be automatically corrected. Spectronaut determines the ideal extraction window dynamically depending on iRT calibration and gradient stability. Qualitative criteria for proteins: Precursor Threshold 1.0\% Q value (FDR), Protein Threshold 1.0\% FDR. Decoy generation was set to mutated which is similar to scrambled but will only apply a random number of AA position swamps $(\min =2$, $\max =$ length $/ 2$ ). The average peak area of the first three peptide segments less than 1.0\% FDR was used to calculate the major group quantities.

\section{Quantitation data analysis}

Statistical analysis including data normalization and relative protein quantification on the DIA dataset was performed using Spectronaut 13 (Biognosys AG, Switzerland). After Welch's ANOVA Test, the proteins with a $\mathrm{p}$ value $<0.05$ and fold change $>1.5$ were considered to be "significantly" regulated in protein quantity.

\section{Bioinformatics analysis}

Blast2GO version 5 was used for functional annotation. GOATOOLS was used to perform GO enrichment analysis. Pathway analysis was performed using KOBAS (http://kobas.cbi.pku.edu.cn/).

\section{Verification of protein by parallel reaction monitoring (PRM)}

Additional quantitative validation of 5 proteins was performed by PRM analysis. Protein preparation was similar as DIA experiment. The peptides were redissolved in solvent A (A: $0.1 \%$ formic acid in water) and carried out in a linear gradient of acetonitrile with $0.1 \%$ formic acid at $400 \mathrm{~nL} / \mathrm{min}$ for $120 \mathrm{~min}$. After that, PRM analysis was performed by on-line nanospray LC-MS/MS on Orbitrap Fusion ${ }^{\mathrm{TM}}$ Lumos $^{\mathrm{TM}}$ Tribrid $^{\mathrm{TM}}$ mass spectrometer coupled to an EASY-nanoLC 1000 system. The settings for PRM were according to the method described by Zhang et al. [12] with some modification. Full MS scans in the mass range from $\mathrm{m} / \mathrm{z} 350$ to 1200 were acquired with a resolution of 120,000 . MS2 spectra were acquired with a resolution of 30,000. The maximum ion injection time and the AGC target were $70 \mathrm{~ms}$ and $2 \mathrm{e}^{5}$, respectively. The inclusion list exported from SpectroDive was imported into the mass list table in the PRM mode. For PRM analysis, raw files of the targeting runs were analyzed in SpectroDive 9.10 with default settings. Q-value cutoff $1 \%$ on precursor was applied. The average of filtered peptides were used to calculate the protein quantities.

\section{Statistical and phylogenetic analyses}

A completely randomized design with three replicates was performed. SPSS software (version 17.0) was used to analyze the data. Significant differences were determined by Duncan's multiple range test at $5 \%$ level.

\section{Results \\ Change in browning index of Thompson seedless grape dry in shade}

The browning index increased with the increase of drying time from day 10 (Fig. 1). The browning index remained stable in the first 8 days. At the end of the shade-drying, the grapes are all browning. The 


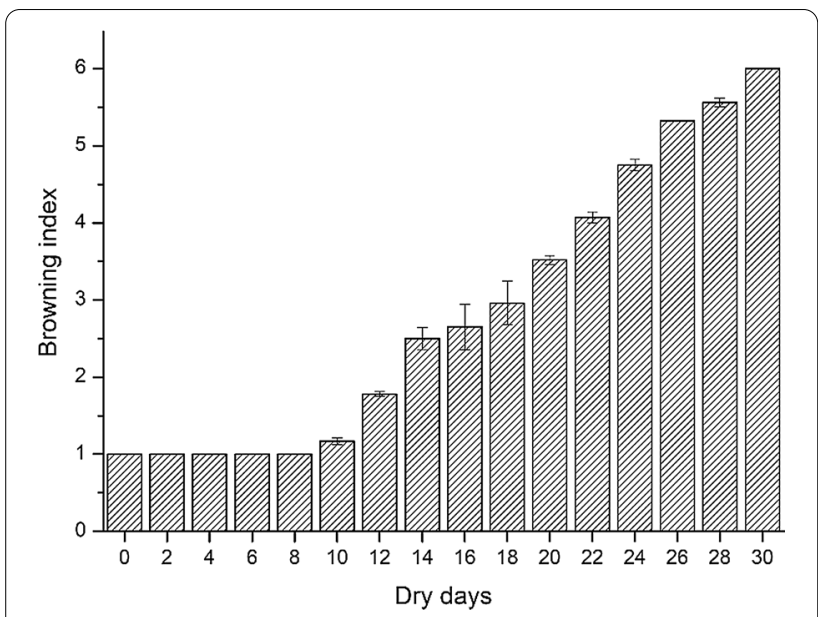

Fig. 1 Changes in browning index of samples. Browning was assessed by measuring the extent of the browned area of 50 individual fruits, the BI was calculated as $\Sigma$ (browning scale $\times$ proportion of corresponding fruits within each class)

browning index of the grapes is 6 , increased by 5 $(P<0.01)$ over fresh grapes. Therefore, fresh grapes and the grapes shade-dried for 30 days were selected for further protein analyses.

\section{Identification and $\mathrm{GO}$ analysis}

In this study, 58,642 precursors were generated. These precursors leading to an identification of 35,803 peptides and 5431 proteins. Proteins with a 1.5 -fold change in protein amount (mean value of all comparison groups) and a $P$-value (t-test of all comparison groups) less than 0.05 were defined as significantly changed proteins (SCP). In all, 739 proteins were identified as SCP. Among these proteins, 550 proteins were up-regulated and 189 proteins were down-regulated.

Go analysis of level 2 was performed on SCP to study their biological functions of Thompson seedless grape in the browning process. As shown in Fig. 2, proteins which play roles in the organic substance metabolic process (17\%), the regulation of molecular function (17\%), the nitrogen compound metabolic process (15\%), the cellular component organization (13\%), response to stress $(6 \%)$, and small molecule metabolic process $(6 \%)$ had been significantly changed in their amount during shade-drying. As shown in Fig. 3, 30\% and 22\% of SCP are related to enzyme regulation and isomerase activity, respectively. The percentage of SCP with molecular functions in lipid binding, oxidoreductase activity, protein binding, and hydrolase activity were calculated to be $13 \%, 12 \%, 5 \%$, and 5\%, respectively. As shown in Fig. 4, 83\% of SCP were enriched in organelle lumen and catalytic complex.

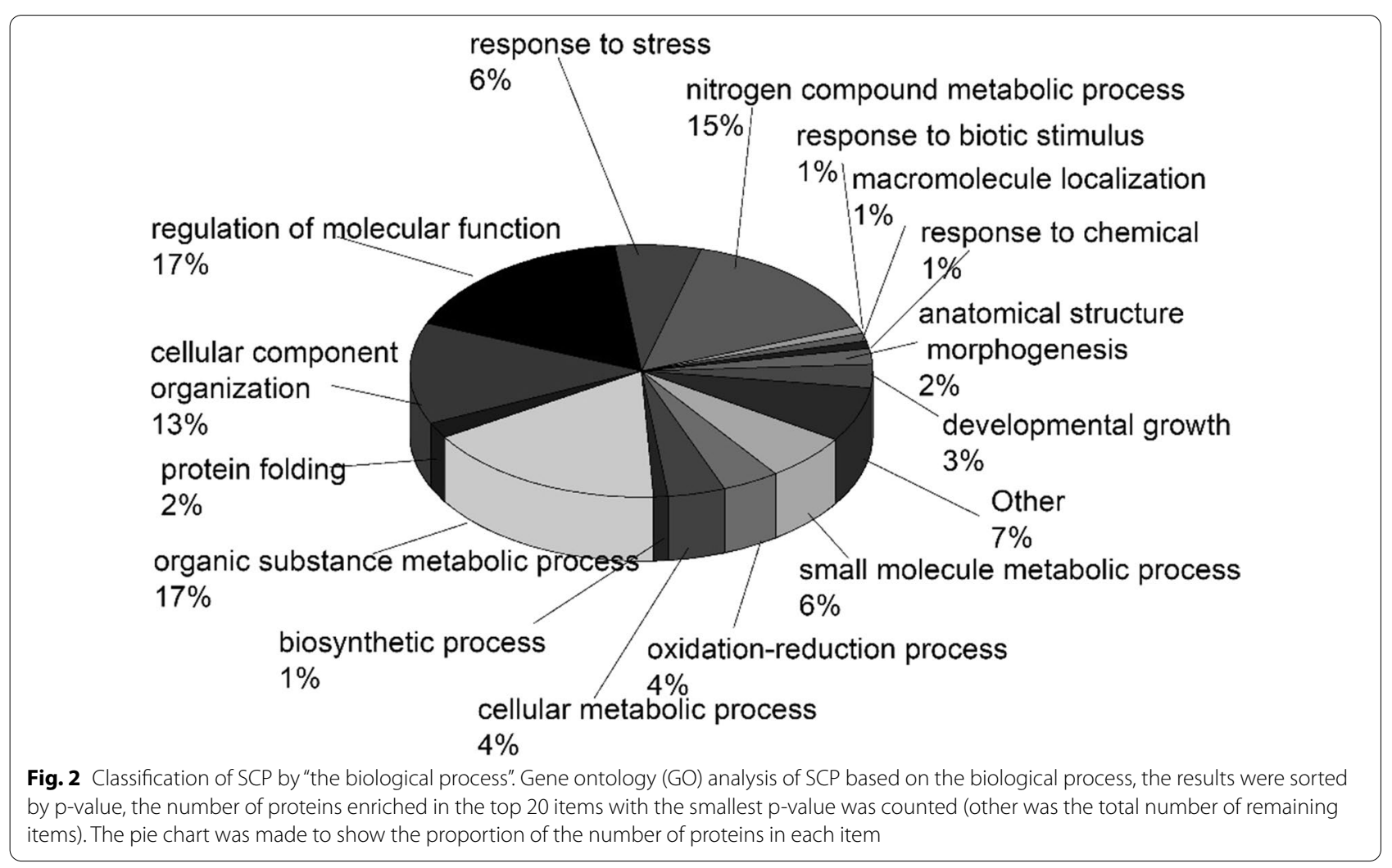




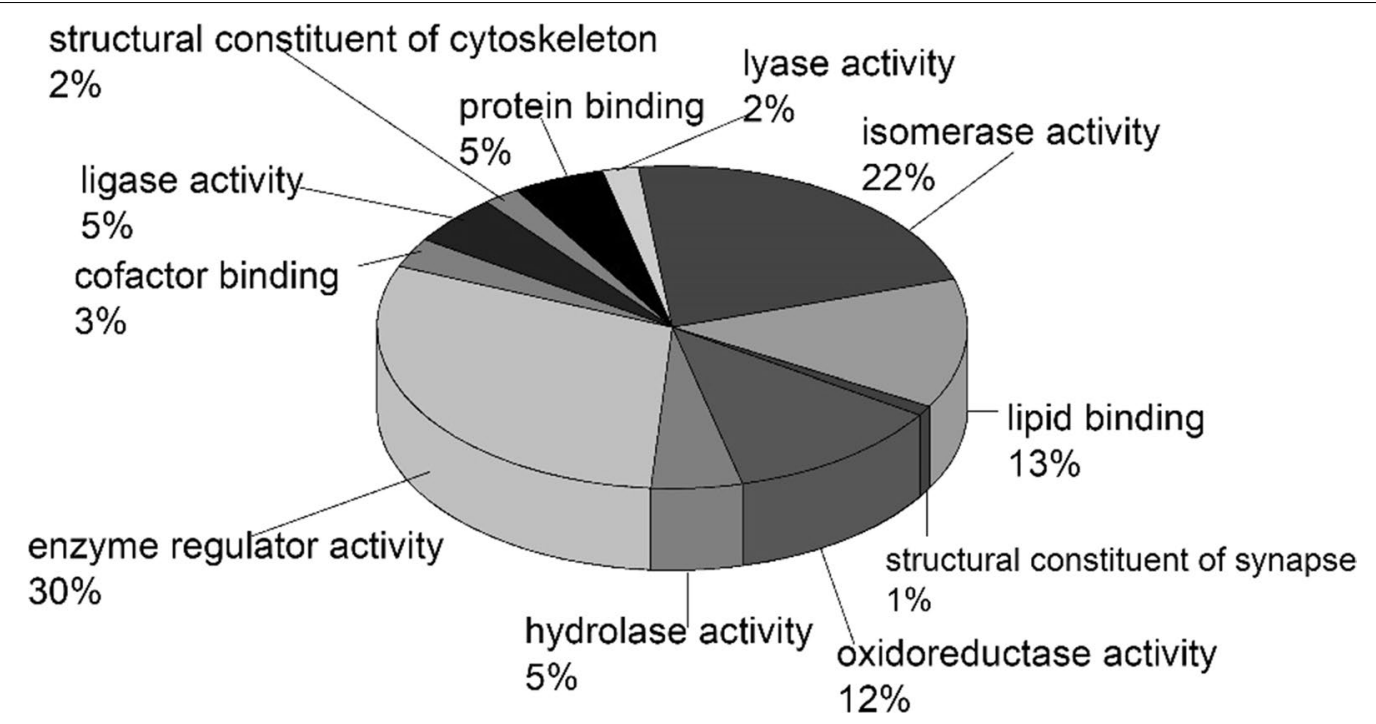

Fig. 3 Classification of SCP by "molecular function". Gene ontology (GO) analysis of SCP based on the molecular function, the results were sorted by $p$-value, the number of proteins enriched in the top 20 items with the smallest p-value was counted (other was the total number of remaining items). The pie chart was made to show the proportion of the number of proteins in each item

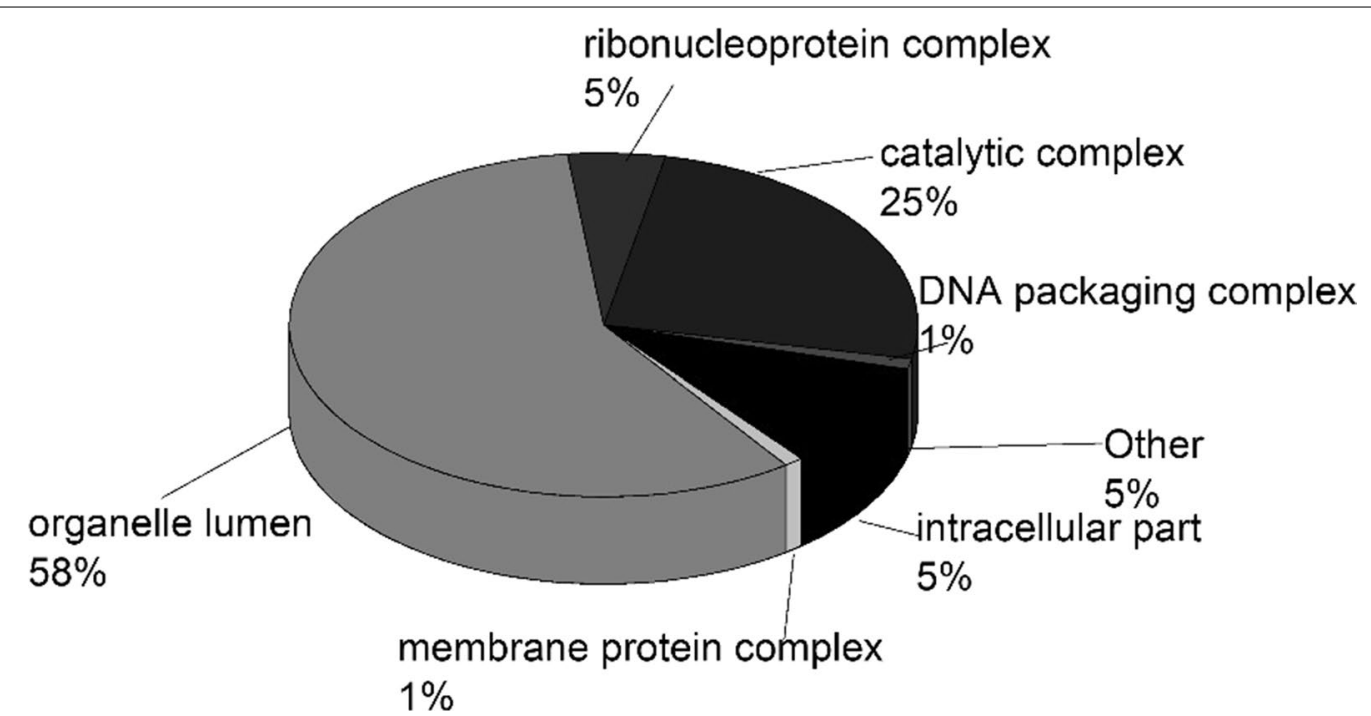

Fig. 4 Classification of SCP by "the cellular component". Gene ontology (GO) analysis of SCP based on the cellular component, the results were sorted by p-value, the number of proteins enriched in the top 20 items with the smallest p-value was counted (other was the total number of remaining items). The pie chart was made to show the proportion of the number of proteins in each item

\section{KEGG pathway analysis of SCP}

Pathway analysis on SCP was performed based on KEGG database. The top 20 SCP-involved pathways with the lowest p-value were shown in Fig. 5. The y axis indicates the percentage of the protein which is involved in the correlated pathway in total SCP.

The results showed that the amount of the proteins which are involved in Metabolic pathways, Biosynthesis of secondary metabolites, Carbon metabolism,
Biosynthesis of amino acids, and Protein processing in endoplasmic reticulum pathways is prone to be affected by shade-drying. Tyrosine metabolism, Fatty acid biosynthesis, and Citrate cycle (TCA cycle) are the key components in grape metabolism pathways. However, we found that many proteins which are involved in other four pathways, Glutathione metabolism, Fatty acid degradation, Phenylalanine metabolism, and Peroxisome, were also significantly changed during the process of shade-drying. 


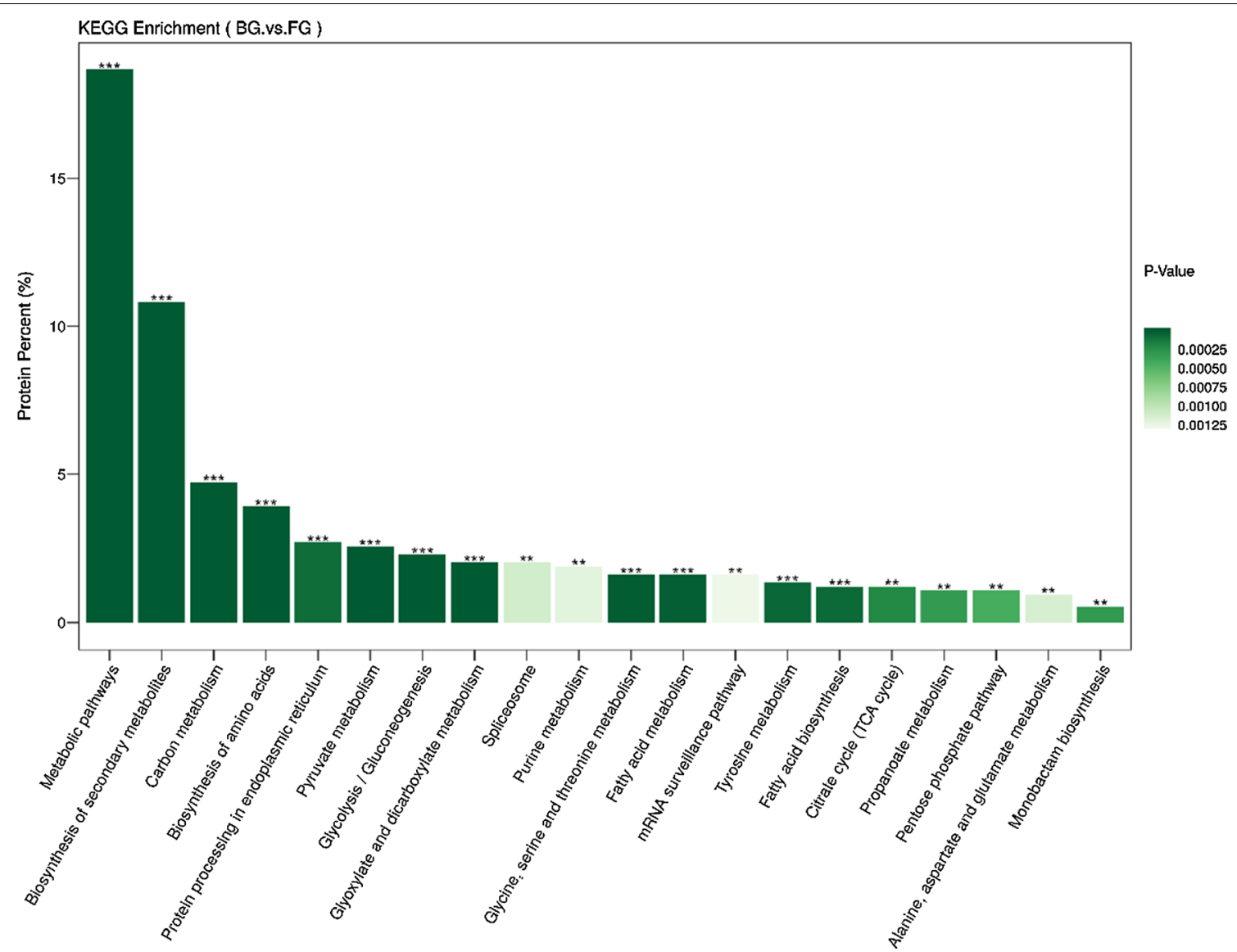

Fig. 5 KEGG enrichment analyses of the differentially expressed proteins (TOP 20). A darker color means a smaller p-value, ${ }^{* *}$ and ${ }^{* *}$ indicated the corrected $p$-value $<0.001$ and $<0.01$, respectively

In addition, the Oxidative phosphorylation, Phenylalanine, tyrosine and tryptophan biosynthesis, Glycerophospholipid metabolism, and Phenylpropanoid biosynthesis pathways may also be largely affected by shade-drying.

\section{The browning-related proteins}

According to their functional description, some SCPs were further classified. As illustrated in Table 1, the grape browning-related proteins are enriched in the phenylpropanoid biosynthesis, tyrosine metabolism, phenylalanine metabolism, oxidative phosphorylation metabolic, Glutathione metabolism, Peroxisome, and Fatty acid degradation pathways. So, the following sections will focus on the involvement of these proteins for the above seven key pathways.

\section{PRM analysis}

PRM has been widely used to detect and quantify target proteins due to its high specificity and sensitivity [13]. To validate the results of DIA, PRM was performed to analyze the five random SCP (four up-regulated:
A5AU08, F6H344, F6H8B4and F6HJJ4, and one downregulated: D7U030) in Table 2. The five proteins are glutathione peroxidase, ATPase, phospholipid hydroperoxide glutathione peroxidase, Oxygen-evolving enhancer protein, and malate dehydrogenase. These proteins are involved in glutathione metabolism, Oxidative phosphorylation, Photosynthesis, and Biosynthesis of secondary metabolites pathway.

The results of PRM analysis revealed that these proteins possess similar expression tendencies (fold change; between BG and FG) with those from proteomic data. For example, the PRM for the protein of phospholipid hydroperoxide glutathione peroxidase was induced by 2.45 -fold, which is similar to the 2.12 fold increase in the protein expression level. The PRM for the protein of ATPase was down-regulated by 1.67fold, which is similar to the 2.28 -fold decrease in the protein expression level.

Although some quantitative differences in the results from the two analytical platforms can be observed, the 
Table 1 The information of proteins related to browning of Thompson seedless grape

\begin{tabular}{|c|c|c|c|c|}
\hline Protein name & Protein accessions & Fold change & Functional description & Pathway names \\
\hline POD & F6GXY7 & 1.71 & Cationic peroxidase 1-like & Phenylpropanoid biosynthesis \\
\hline Beta-glucosidase & D7U288 & 2.01 & Beta-glucosidase 11 & Phenylpropanoid biosynthesis \\
\hline Acylpyruvase FAHD1 & D7SYB8 & 1.56 & Acylpyruvase FAHD1 & Tyrosine metabolism \\
\hline Aspartate aminotransferase & D7SUD7 & 1.65 & Aspartate aminotransferase & Phenylalanine metabolism \\
\hline Fumarylacetoacetase & D7TRJ7 & 1.54 & Fumarylacetoacetase & Tyrosine metabolism \\
\hline Primary amine oxidase & F6HUI4 & 1.72 & Primary amine oxidase-like & Tyrosine metabolism \\
\hline Alcohol dehydrogenase & F6H098 & 1.51 & Alcohol dehydrogenase & Tyrosine metabolism \\
\hline Alcohol dehydrogenase & EOCQN2 & 1.96 & Alcohol dehydrogenase I & Tyrosine metabolism \\
\hline Bifunctional aminotransferase & D7SW04 & 1.57 & $\begin{array}{l}\text { Bifunctional aspartate aminotransferase and glu- } \\
\text { tamate/aspartate-prephenate aminotransferase }\end{array}$ & Tyrosine metabolism \\
\hline ATP synthase & D7SM33 & -1.66 & ATP synthase subunit delta' & Oxidative phosphorylation \\
\hline ATPase & D7U030 & -1.67 & ATPase, F0 complex, subunit G & Oxidative phosphorylation \\
\hline SOD & D7SNA2 & 1.88 & Superoxide dismutase $[\mathrm{Cu}-\mathrm{Zn}]$ & Peroxisome \\
\hline SOD & F6HTY5 & 2.02 & Superoxide dismutase $[\mathrm{Cu}-\mathrm{Zn}]$ & Peroxisome \\
\hline SOD & F6HTX9 & 2.99 & Superoxide dismutase $[\mathrm{Cu}-\mathrm{Zn}]$ & Peroxisome \\
\hline CAT & D7UD99 & 1.52 & Catalase isozyme 1 & Peroxisome \\
\hline GSH & A5AU08 & 2.85 & Glutathione peroxidase & Glutathione metabolism \\
\hline GSH & $\mathrm{F} 6 \mathrm{H} 344$ & 2.12 & $\begin{array}{l}\text { Phospholipid hydroperoxide glutathione peroxi- } \\
\text { dase } 1\end{array}$ & Glutathione metabolism \\
\hline Acyl-coenzyme A oxidase 3 & $\mathrm{~F} 6 \mathrm{H} 4 \times 3$ & 2.03 & Acyl-coenzyme A oxidase 3 & Fatty acid degradation \\
\hline Aldehyde dehydrogenase & D7SP43 & 2.91 & Aldehyde dehydrogenase family 3 member F1 & Fatty acid degradation \\
\hline Alcohol dehydrogenase & F6H098 & 1.51 & Alcohol dehydrogenase & Fatty acid degradation \\
\hline Alcohol dehydrogenase & EOCQN2 & 1.96 & Alcohol dehydrogenase I & Fatty acid degradation \\
\hline Acetyl-CoA acetyltransferase & F6HRX1 & 1.63 & Acetyl-CoA acetyltransferase & Fatty acid degradation \\
\hline
\end{tabular}

Table 2 PRM analysis on SCP

\begin{tabular}{llccr}
\hline & Protein & BG/FG ratio & P value & BG/FG ratio (DIA) \\
\hline A5AU08 & Glutathione peroxidase & 1.40 & 0.035 & 2.85 \\
D7U030 & ATPase & -2.28 & $2.99 E-06$ & -1.67 \\
F6H344 & Phospholipid hydroperoxide glutathione peroxi- & 2.45 & 0.008 & 2.12 \\
& dase & & & 5.88 \\
F6H8B4 & Oxygen-evolving enhancer protein & 2.40 & 0.001 & 2.38 \\
\hline
\end{tabular}

5 SCP exhibiting similar trend in the PRM results supported the reliability of the DIA data.

\section{Discussion}

Enzymatic browning is a reaction that affects the appearance, quality, and sensory properties of fruits and other vegetables. It is appealing more and more attentions from industry and researchers [14]. The browning of Thompson seedless grape during drying in the barn has been well-acknowledged as the primary factor affecting the development of the raisin industry in Xinjiang, China. The browning of grapes only occurred in the middle and late stage of the shade-drying. After 10 days of drying in shade, the browning dramatically developed. The shade-drying process is a dehydration stress for grapes. Dehydration not only reduces the weight of the grapes, but also causes changes in the metabolism and compounds of the grapes. This process involves the expression of many different proteins. Therefore, fresh and completely browning Thompson seedless grapes were used as research material in this study. DIA sequencing technology was used to determine the protein changes in the process of shade drying of Thompson seedless grapes. The main regulatory proteins which are related 
to browning were screened. Changes in their metabolic pathways were also analyzed. PRM was applied to support the analyses on the expression of proteins. This study laid a theoretical foundation to the study of enzymatic browning of Thompson seedless grapes during shade drying. In addition, our results provided a guidance for the effective control of browning in industry.

In this study, differential proteomics analysis was carried out using fresh grapes and browning grapes as materials. In all, 739 proteins were identified as SCP. Among these proteins, the amount of 550 proteins were upregulated, while the amount of 189 proteins were downregulated. The results of Gene Ontology annotation showed that the SCP are mainly involved in the organic substance metabolic process, regulation of molecular function, nitrogen compound metabolic process, cellular component organization, response to stress, and small molecule metabolic process. The KEGG results indicate that between BG and FG, the Metabolic pathways, Biosynthesis of secondary metabolites, Carbon metabolism, Biosynthesis of amino acids, and Protein processing in endoplasmic reticulum pathways were the most affected pathways during the drying process.

Previous studies found that the cause of grape browning is the enzymatic reaction $[15,16]$. Enzymatic browning is caused by structural damage to the fruit tissue and it is originated by enzymes, such as the PPO or POD. These enzymes react with phenolic substrates to produce quinones, which can be further oxidized and polymerized to form darker pigments (brown) on the surface of fruits $[17,18]$. POD are the main enzymes involved in the phenolic oxidation of many fruits. In our research, the POD (F6GXY7) is 1.71-fold up-regulated in the BG. POD is the terminal enzyme of phenylpropanoid metabolic pathway involved in lignin synthesis in plants.

The phenols are main substrates of enzymatic browning, which are closely related to browning [19]. Hogan et al. [20] considered that the browning degree of grape had a high correlation with the contents of total phenol, proanthocyanidins, catechins and root skin glycosides. Grncarevic et al. [10] studied the relationship between phenolic compounds and browning during the drying of grapes. It is acknowledged that browning is related to the production of medium and low molecular weight polymers. The enzymatic oxidative degradation of hydroxycinnamate and flavan-3-ol derivatives during drying is an important cause of grape browning. Singleton pointed out that the caffeic acid disappeared during the drying process of the Thompson seedless grape, but no $s$-glutathionyl caffeic acid production was detected. Durst et al. [21] found that flavanol compounds were less sensitive to enzymatic browning than hydroxy cinnamic acid compounds (cinnamic acid, caffeic acid, p-coumaric acid, ferulic acid, etc.), but the degradation of proanthocyanidins and flavane-3-ols was completely during the drying process. Polyphenols are mainly derived from phenylpropanoid metabolic pathway [22]. In our study, coumarin was produced as a result of the reaction between the beta-glucosidase (D7U288) and $\beta$-Dglucosyl-2-coumarinate. Its amount was significantly increased (twofold) during shade-drying. The amount of peroxidase (F6GXY7) which catalyze the last step of the phenylpropanoid pathway for the production of lignin, was 1.71-fold increased in the drying process. These two proteins are involved in the Phenylpropanoid biosynthesis. Besides, Tyrosine and phenylalanine are the precursors of phenolic compounds in fruit. In our study, we found that the tyrosine metabolism and phenylalanine metabolism were favored in the shade-drying of grapes. In tyrosine metabolism, the presence of seven proteins (D7SYB8, D7SUD7, D7TRJ7, F6HUI4, F6H098, E0CQN2, D7SW04) were significantly up-regulated. Among these proteins, the amount of aspartate aminotransferase (D7SUD7) which could turn 4-hydroxy-phenylpyruvate into tyrosine was increased by 1.65 -fold. In Phenylalanine metabolism, aspartate aminotransferase (D7SUD7) could convert phenylpyruvate into phenylalanine. Hence, the key proteins aspartate aminotransferase significantly upregulated during grape drying, resulting in an enhancement in the syntheses of tyrosine and phenylalanine. The up-regulated proteins in the Phenylpropanoid biosynthesis and the precursor substances of phenols are important factors related to the browning of grape.

Normally, the PPO and phenolic compounds are separated in different cell compartments. Studies have shown that the integrity of cell membrane system is closely related to energy [23-26]. The change of energy level in cells mainly comes from energy metabolism. In the energy metabolism pathway, oxidative phosphorylation occurs on the inner membrane of mitochondria, which is the third stage of respiratory metabolism and could produce $95 \%$ energy in cells [27]. In this study, the oxidative phosphorylation pathway of browned grapes was changed, and consequently affects the energy metabolism of grapes. In the oxidative phosphorylation metabolic pathway, the synthesis of two ATP synthase, D7SM33 and D7U030, was down-regulated by 1.66 -fold and 1.77 -fold, respectively. In fact, there is a direct relationship between ATP synthesis and membrane degradation during the browning of fruits [28]. Energy is an essential element to maintain the structure and biochemical functions of the cell membrane system. Shortage of energy may lead to a decrease of cell membrane lipid synthesis rate and the aggravation of cell membrane lipid peroxidation, which will cause the imbalance of cell membrane lipid metabolism and subsequently leads to browning [29]. 
In recent years, it has been found that the enzymatic browning of fruit is closely related to the enhancement of membrane lipid peroxidation and the destruction of membrane structure caused by the imbalance of reactive oxygen metabolism [30]. Excessive accumulation of reactive oxygen species is the main cause of membrane lipid peroxidation. The free radical theory suggests that superoxide dismutase (SOD) scavenge superoxide anions through disproportionation reactions to generate nontoxic $\mathrm{O}_{2}$ and $\mathrm{H}_{2} \mathrm{O}_{2}, \mathrm{H}_{2} \mathrm{O}_{2}$ that can be reduced to $\mathrm{H}_{2} \mathrm{O}$ by catalase (CAT) or ascorbate peroxidase (APX), thereby reducing the accumulation of reactive oxygen species (ROS) such as superoxide anion and $\mathrm{H}_{2} \mathrm{O}_{2}$ and reducing the destruction of cell membranes. Glutathione (GSH) is the major enzyme that catalyzes the oxidation of reduced glutathione in the glutathione redox cycle, which could specifically catalyze the reaction of reduced glutathione with ROS to produce oxidized glutathione (GSSG), thus protecting the biofilm from ROS damage and maintaining the normal function of cells during stress. In this study, ROS was accumulated in the shade-dry process of grape because the production and clearance system of ROS was broken after the grapes suffered with dehydration stress. The syntheses of SOD, CAT and GSH protein in browning grape are significantly up-regulated. This may result from the fact that grape reduces oxidative stress by regulating antioxidant enzymes in the body, which is a response of plants to oxidative stress [31, 32]. Phenolic compounds can scavenge excess ROS in the fruit, but the resulting brown-colored oxidation products are also one of the causes of fruit browning [33].

Drying is a dehydration stress for grapes. When plants are subjected to stress, the first site of injury is the cell membrane [34]. Fatty acids are the main components of the cell membrane of organisms. When the metabolism of ROS is dysregulated, the membrane lipid peroxidation intensifies. As a result, unsaturated fatty acids in the phospholipid bimolecular layer in the membrane are oxidized and decomposed, resulting in the destruction of the whole membrane $[35,36]$ and eventually browning of fruits $[37,38]$. In this study, the fatty acid degradation pathway was favored, where acyl-coenzyme oxidase (3F6H4X3) is 2.05-fold up-regulated. It can catalyze the first step of $\beta$-fatty acid oxidation, and it is the rate-limiting enzyme for $\beta$-oxidation $[39,40]$. In addition, 4 proteins (D7SP43, F6H098, E0CQN2, F6HRX1) which are involved in the fatty acid catabolism were up-regulated. D7SP43 synthesis was 2.91-fold upregulated, F6H098 and E0CQN2 was 1.51-fold and 1.96-fold up-regulated, respectively. Aldehyde dehydrogenase can convert fatty acids into aldehyde and then into alcohol. F6HRX1 (acetyl-CoA acetyltransferase) localized in the peroxisome and is modulated 1.63 -fold in fatty acid degradation. It can catalyze the conversion of acyl-coenzyme $\mathrm{A}$ and acetyl-coenzyme A to 3-oxy-coenzyme A in fatty acid degradation pathway. In summary, the synthesis of multiple key proteins in fatty acid degradation pathway is up-regulated, which promotes the catabolism of fatty acids in grapes. Thus, the membrane lipid metabolism and integrity of grape cells are affected, and thereby causing the grapes browning.

\section{Acknowledgements \\ Not applicable.}

\section{Authors' contributions}

All authors designed the experiments together, and FJL, WSH, ZSF and YJB conducted the experiments. FLL, YXT, YYF, WZH, XLL, XTF and CW analyzed and interpreted the results, and prepared the manuscript. All authors read and approved the final manuscript.

\section{Funding}

This work was supported by the National Natural Science Foundation of China (Nos. 31660466 and 31201448), the plan of Introduction 100 young doctors in Xinjiang, and the technical missions for quality and safety of agricultural products (No. CAQS-2020-05-13).

\section{Availability of data and materials}

The datasets used and analysed during the current study are available from the corresponding author on reasonable request.

\section{Declarations}

\section{Competing interests}

The authors declare that they have no competing interests.

\section{Author details}

${ }^{1}$ Institute of Quality Standards \& Testing Technology for Agro-Products, Xinjiang Academy of Agricultural Sciences, Key Laboratory of Agro-Products Quality and Safety of Xinjiang, Laboratory of Quality and Safety Risk Assessment for Agro-Products (Urumqi), Ministry of Agriculture and Rural Affairs, Urumqi 830091, China. ${ }^{2}$ College of Food Science and Pharmacy, Xinjiang Agricultural University, Urumqi 830052, China. ${ }^{3}$ Management of Scientific Research, Xinjiang Academy of Agricultural Sciences, Urumqi 830091, China.

Received: 14 January 2021 Accepted: 4 May 2021

Published online: 11 May 2021

\section{References}

1. Rees D, Farrell G, Orchard J (2012) Crop post-harvest: science and technology. Wiley-Blackwell, Oxford

2. Jayaprakasha GK, Girennavar B, Patil BS (2008) Radical scavenging activities of Rio Red grapefruits and Sour orange fruit extracts in different in vitro model systems. Bioresour Technol 99(10):4484-4494

3. Fang YL, Zhang A, Wang H, Li H, Zhang ZW, Chen SX, Luan LY (2010) Health risk assessment of trace elements in Chinese raisins produced in Xinjiang province. Food Control 21(5):732-739

4. Mayer AM, Harel E (1979) Polyphenol oxidases in plants. Phytochemistry 18(2):193-215

5. Lin YF, Lin HT, Lin YX, Zhang S, Chen YH, Jiang XJ (2016) The roles of metabolism of membrane lipids and phenolics in hydrogen peroxideinduced pericarp browning of harvested longan fruit. Postharvest Biol Technol 2016:111

6. Sheng L, Zhou X, Liu ZY, Wang JW, Zhou Q, Wang L et al (2016) Changed activities of enzymes crucial to membrane lipid metabolism accompany pericarp browning in 'Nanguo' pears during refrigeration and subsequent shelf life at room temperature. Postharvest Biol Technol 117:1-8 
7. Antelmi G, Bellincontro A, Mencarelli F, Nicoletti I, Corradini D (2010) How dehydration temperature and weight loss affect the biosynthesis of nutritional compounds in irrigated 'Aleatico' grape. Acta Hortic 877:693-698

8. Mencarelli F, Bellincontro A, Nicoletti I, Cirilli M, Muleo R, Corradini D (2010) Chemical and biochemical change of healthy phenolic fractions in winegrape by means of postharvest dehydration. J Agric Food Chem 58(13):7557-7564

9. Bonghi C, Rizzini FM, Gambuti A, Moio L, Chkaiban L, Tonutti P (2012) Phenol compound metabolism and gene expression in the skin of wine grape (Vitis vinifera L.) berries subjected to partial postharvest dehydration. Postharvest Biol Technol 67:102-109

10. Serratosa MP, Lopez-Toledano A, Merida J, Medina M (2008) Changes in color and phenolic compounds during the raisining of grape Cv. Pedro Ximenez. J Agric Food Chem 56(8):2810-2816

11. Lin HT, Chen L, Lin YF, Jiang YM (2010) Fruit weight loss and pericarp water loss of harvested longan fruit in relation to pericarp browning. Acta Hortic 863:587-592

12. Zhang R, He Y, Yi J, Zhang LJ, Shen CP, Liu SJ et al (2019) Proteomic and metabolic elucidation of solar-powered biomanufacturing by bio-abiotic hybrid system. Chem 6(1):234-249

13. Zhang J, Li QR, Liu JH, Lu YH, Wang Y, Wang YH (2020) Astaxanthin overproduction and proteomic analysis of Phaffia rhodozyma under the oxidative stress induced by $\mathrm{TiO}_{2}$. Bioresour Technol 311:123525

14. Soliva-Fortuny RC, Elez-Martínez P, Sebastián-Calderó M, Martín-Belloso $O$ (2002) Kinetics of polyphenol oxidase activity inhibition and browning of avocado purée preserved by combined methods. J Food Eng 55(2):131-137

15. Grncarevic M, Hawker JS (1971) Browning of sultana grape berries during drying. J Sci Food Agric 22(5):270-272

16. Lichter A, Kaplunov T, Zutahy Y, Daus A, Alchanatis V, Ostrovsky V et al (2011) Physical and visual properties of grape rachis as affected by water vapor pressure deficit. Postharvest Biol Technol 59(1):25-33

17. Quevedo R, Valencia E, Alvarado F (2013) Comparison of whiteness index vs. fractal Fourier in the determination of bloom chocolate using image analysis. Food Bioprocess Technol 6(7):1878-1884

18. Song Y, Yao YX, Zhai H, Du YP, Chen F, Wei SW (2007) Polyphenolic compound and the degree of browning in processing apple varieties. Agric Sci China 6(5):607-612

19. Sukhonthara S, Kaewka K, Theerakulkait C (2016) Inhibitory effect of rice bran extracts and its phenolic compounds on polyphenol oxidase activity and browning in potato and apple puree. Food Chem 190:922

20. Hogan S, Zhang L, Li JR, Zoecklein B, Zhou KQ (2009) Antioxidant properties and bioactive components of Norton (Vitis aestivalis) and Cabernet Franc (Vitis vinifera) wine grapes. LWT Food Sci Technol 42(7):1755

21. Durst RW, Wrolstad RE, Karadeniz F (2000) Polyphenolic composition of raisins. J Agric Food Chem 48(11):5343-5350

22. Strack D (1997) Phenolic metabolism. Academic Press, London

23. Veltman RH, Lenthéric I, Plas LH, Peppelenbos HW (2003) Internal browning in pear fruit (Pyrus communis L. cv Conference) may be a result of a limited availability of energy and antioxidants. Postharvest Biol Technol 28(2):295-302

24. Li PY, Zheng XL, Liu Y, Zhu YY (2014) Pre-storage application of oxalic acid alleviates chilling injury in mango fruit by modulating proline metabolism and energy status under chilling stress. Food Chem 142:72-78
25. Zhang S, Lin HT, Lin MS, Lin YF, Chen YH, Wang H et al (2019) Lasiodiplodia theobromae (Pat.) Griff. \& Maubl. reduced energy status and ATPase activity and its relation to disease development and pericarp browning of harvested longan fruit. Food Chem 275:239-245

26. Lin YF, Lin YX, Lin HT, Chen YH, Wang H, Shi J (2018) Application of propyl gallate alleviates pericarp browning in harvested longan fruit by modulating metabolisms of respiration and energy. Food Chem 240:863-869

27. Qin GZ, Wang Q, Liu J, Li BQ, Tian SP (2010) Proteomic analysis of changes in mitochondrial protein expression during fruit senescence. Proteomics 9(17):4241-4253

28. Zhang ZK, Hu MJ, Yun Z, Wang JB, Feng G, Gao ZY et al (2017) Effect of tea seed oil treatment on browning of litchi fruit in relation to energy status and metabolism. Postharvest Biol Technol 132:97-104

29. Liu H, Song LL, You YL, Li YB, Duan XW, Jiang YM et al (2011) Cold storage duration affects litchi fruit quality, membrane permeability, enzyme activities and energy charge during shelf time at ambient temperature. Postharvest Biol Technol 60(1):24-30

30. Jiang YM, Li YB (2003) Effects of low-temperature acclimation on browning of litchi fruit in relation to shelf life. J Pomol Hortic Sci 78(4):437-440

31. Breusegem FV, Vranová E, Dat JF, Inzé D (2001) The role of active oxygen species in plant signal transduction. Plant Sci 161(3):405-414

32. Liu Y, Xiong Y, Bassham DC (2009) Autophagy is required for tolerance of drought and salt stress in plants. Autophagy 5(7):954-963

33. Wang JW, Zhou X, Zhou Q, Liu ZY, Sheng L, Wang L et al (2017) Proteomic analysis of peel browning of 'Nanguo' pears after low-temperature storage. J Sci Food Agric 97(8):2460-2467

34. Lin YX, Lin HT, Chen YH, Wang H, Ritenour MA, Lin YF (2019) Hydrogen peroxide-induced changes in activities of membrane lipidsdegrading enzymes and contents of membrane lipids composition in relation to pulp breakdown of longan fruit during storage. Food Chem 297:124955

35. Jaleel CA, Manivannan P, Sankar B, Kishorekumar A, Panneerselvam R (2007) Calcium chloride effects on salinity-induced oxidative stress, proline metabolism and indole alkaloid accumulation in Catharanthus roseus. CR Biol 330(9):674-683

36. Elkahoui S, Hernández JA, Abdelly C, Ghrir R, Limam F (2005) Effects of salt on lipid peroxidation and antioxidant enzyme activities of Catharanthus roseus suspension cells. Plant Sci 168(3):607-613

37. Jiang YM, Duan XW, Joyce D, Zhang ZQ, Li JR (2004) Advances in understanding of enzymatic browning in harvested litchi fruit. Food Chem 88(3):443-446

38. Saquet AA, Streif J, Bangerth F (2003) Energy metabolism and membrane lipid alterations in relation to brown heart development in 'Conference' pears during delayed controlled atmosphere storage. Postharvest Biol Technol 30(2):123-132

39. Arent S, Pye VE, Henriksen A (2008) Structure and function of plant acylCoA oxidases. Plant Physiol Biochem 46(3):292-301

40. Kawaguchi A, Tsubotani S, Seyama Y, Yamakawa T, Osumi T, Hashimoto A et al (1980) Stereochemistry of dehydrogenation catalyzed by acyl-CoA oxidase. J Biochem 5:1481-1486

\section{Publisher's Note}

Springer Nature remains neutral with regard to jurisdictional claims in published maps and institutional affiliations. 